\title{
ANALYSE DES PROPOS TENUS PAR DES REFUGIÉS MINEURS NON ACCOMPAGNÉS DANS DES TEXTES IDENTITAIRES. NOUVEAUX VECTEURS D'INCLUSION ?
}

\author{
An analysis of identity text entries by unaccompanied \\ refugee minors. New ways to support inclusion?
}

According to EKKA (National Center of Social Solidarity), in 2016, almost 5000 unaccompanied minor refugees were registered in Greece. Our research is part of a research for new methodological tools enabling language teachers to adapt their teaching to the needs of these new learners, in particular by exploiting their cultural and linguistic capital. One of these tools is the "identity text" which, according to Cummins (2006), is a reflection of the author's identity at some point in his/her biography. The hypotheses of our research are that the production of identity texts by unaccompanied minor refugees can reinforce $(\mathrm{H} 1)$ their self-esteem, (H2) their motivation for learning new languages in school and (H3) the development of skills that could lead to better integration (affective, linguistic, cultural and socio-cultural) within the host country's school system. These hypotheses are confirmed by the results of cross-content analysis of a collection of identity texts produced online (on Facebook) in 2016-2017 by 30 unaccompanied minor refugees of Syrian, Afghan and Pakistani origin, aged between 14 to 18 years old. It appears that our three hypotheses are validated to a significant extent. These findings may be an incentive for language teachers, but perhaps also for the actors of Formal and Non Formal education to observe and anchor the linguistic and cultural skills of newly arrived refugees, so that they find their place more easily in a welcoming society. 
Keywords: identity texts, unaccompanied minors refugees, plurilingual identity, integration

Słowa kluczowe: narracje tożsamościowe, nieletni uchodźcy bez opieki, tożsamość różnojęzyczna, integracja

\section{Introduction}

La crise des réfugiés s'est traduite par une augmentation considérable du nombre des réfugiés " mineurs non accompagnés ", de ces enfants ou adolescents qui arrivent dans un pays en quête de protection internationale, sans famille ni quelque autre accompagnateur adulte. Selon le Centre national pour la solidarité sociale, de janvier 2016 à novembre 2017, 10175 mineurs non accompagnés ont été enregistrés en Grèce (Haut-Commissariat des Nations unies pour les réfugiés, $2017: 1$ ). La plupart d'entre eux viennent de la Syrie, de I'Afghanistan, de I'Irak ou du Pakistan (Id. : 3). Ces enfants non accompagnés sont détenus dans des conditions inadéquates, le plus souvent dans des installations fermées, ou en garde à vue par la police pendant de longues périodes, faute de logement stable et sûr, à court ou à long terme (Rapport collectif de Act!onaid et al., 2016 : 4). Actuellement, davantage d'abris de transit et de longue durée ont été créés par des Organisations non gouvernementales, avec la collaboration du ministère du Travail. Mais le transfert des enfants dans ces structures est retardé, en raison de leur surpeuplement actuel. L'éducation de la majorité de ces enfants a été perturbée pendant de très longues périodes, par la durée de leurs voyages et par le temps passé à attendre en Grèce.

Aucune inscription systématique des élèves dans les écoles n'est réalisée et les élèves qui fréquentent malgré tout l'école grecque sont confrontés à des difficultés considérables, en raison de l'absence de mise en place de programmes d'études interculturels et d'approches pédagogiques inclusives. Les enfants non accompagnés qui se trouvent en détention dans des installations fermées ont un accès aux loisirs et à l'éducation mais les enfants non accompagnés détenus dans les postes de police $n$ 'ont pas ces possibilités.

Les camps qui disposent d'espaces d'apprentissage dédiés sont rares (Id. : 5). Les enfants résidant dans des refuges participent à des activités éducatives et reçoivent notamment des cours de langue, principalement dispensés en grec, mais aussi en anglais, en français et en allemand. Aucun de ces cours ne se fait dans leurs langues d'origine. Les mineurs non accompagnés s'expriment en arabe, en farsi, en ourdou ou dans une combinaison de ces langues. Leur compétence linguistique n'est pas nécessairement associée à 
leur origine ethnique : elle peut être associée aussi à leur pays de socialisation. Par conséquent, ils utilisent des répertoires linguistiques liés à un ou plusieurs langages, registres ou genres.

Cette situation conduit les chercheurs à mettre au point de nouveaux outils méthodologiques permettant aux enseignants des langues d'adapter leur enseignement aux besoins de ces primo arrivants, en se concentrant sur l'exploitation de leur capital culturel et linguistique. Un de ces outils est le « texte identitaire ».

Cummins (2006) utilise le terme de textes identitaires pour décrire :

« Les produits du travail de création ou des performances créatives d'élèves, réalisé au sein de l'espace pédagogique et orchestré par le professeur. Les étudiants investissent leur identité dans la création de ces textes qui peuvent être écrits, parlés, visuels, musicaux, joués ou constituer des combinaisons multimodales. Le texte identitaire tient alors lieu de miroir dans lequel se reflètent leurs identités sous un jour positif. » ${ }^{1}$

Le texte identitaire constitue donc un reflet de l'identité de son auteur, à un moment donné de sa biographie. II permet de découvrir des moments difficiles de sa biographie qui peuvent influencer / avoir influencé son apprentissage. Ainsi, par le biais des textes identitaires, enseignants et chercheurs tentent de donner une voix aux réfugiés, de faire remonter à la surface la complexité de leur identité et leurs répertoires linguistiques.

On peut rapprocher ces notions de " textes identitaires " et d' " identités " de celle des " récits de vie " introduite par Deprez en 2006. Deprez montre que ces textes permettent de mieux connaître la vie du nouvel arrivé par le biais de ses productions écrites, de lui faire effectuer le récit de moments difficiles d'une façon moins directe. Pour Deprez, les récits de vie constituent un outil permettant de connaître l'auteur au travers d'une littératie réalisée dans sa première langue et d'utiliser justement cette littératie pour l'apprentissage d'une nouvelle langue.

L'usage du texte identitaire permet notamment aux élèves de mettre en valeur leur profil et, en particulier, leur identité plurilingue. Durant ces dernières années, il a constitué un outil de recherche. Ainsi, dans des classes de l'école primaire et dans le cadre d'un cours de langue française, nous avons

\footnotetext{
${ }^{1}$ Cummins et al (2006) Trad. de l'anglais « [...] the products of students' creative work or performances carried out within the pedagogical space orchestrated by the classroom teacher. Students invest their identities in the creation of these texts which can be written, spoken, signed, visual, musical, dramatic, or combinations in multimodal form. The identity text then holds a mirror up to students in which their identities are reflected back in a positive light $»$.
} 
fait produire par les enfants de familles migrantes un grand nombre de textes identitaires (Cf. Auteur, 2009a, 2009b, 2010, 2011 ; Auteur en collaboration, 2010 ; Auteur en collaboration, 2013). Il s'agit de textes dans lesquels les élèves sont appelés à exprimer leurs attitudes envers les autres, leur expérience dans l'apprentissage, non seulement du français mais aussi d'autres matières. Cet outil nous a aidés à découvrir le bagage linguistique des apprenants et à appliquer, par exemple, "le principe de l'interdépendance » et/ou d'autres didactiques du plurilinguisme (Auteur, 2017)

Ces utilisations qui peuvent être faites du texte identitaire apparaissent clairement dans un texte produit dans un camp pour réfugiés mineurs non accompagnés, dont nous livrons la reproduction (Extrait 1) et la traduction ci-dessous.

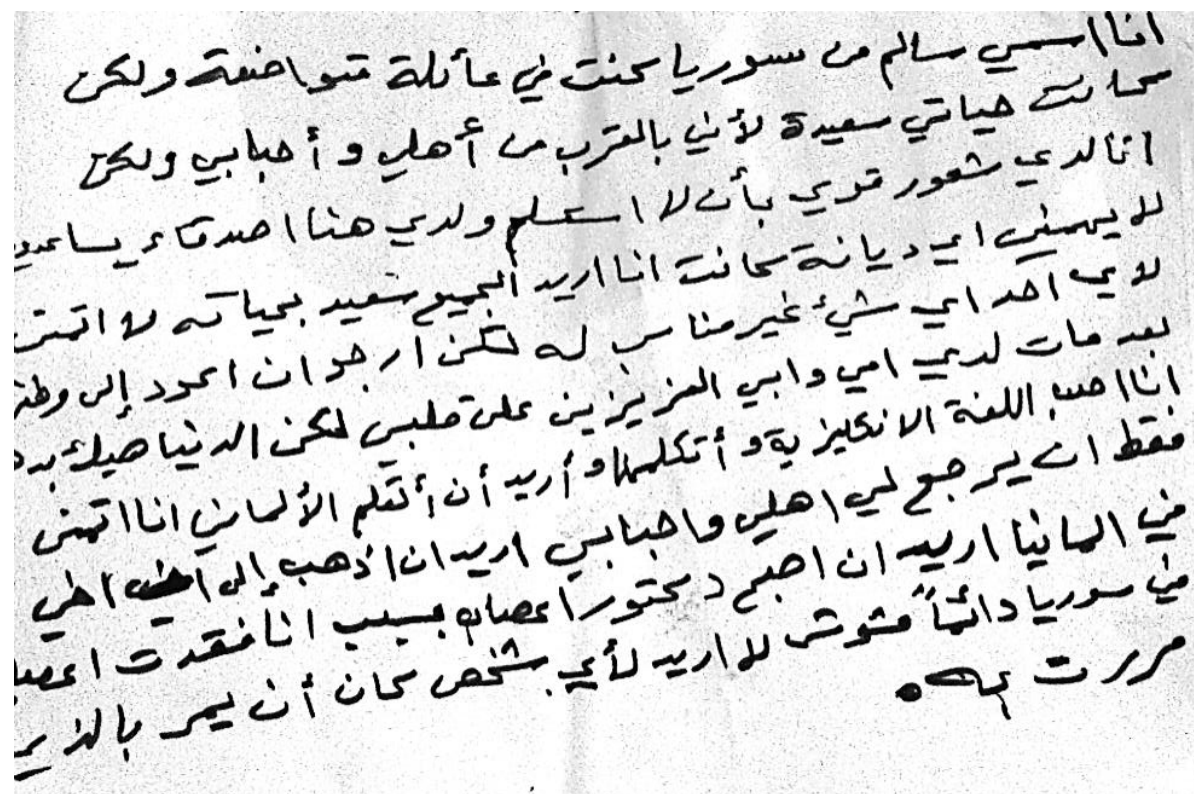

Je m'appelle Salem de Syrie. Je suis issu d'une famille simple. J'avais une vie heureuse parce que j'étais auprès de mes parents et des personnes que j'aime. J'ai une volonté très forte, celle de ne pas laisser tomber. Ici, j'ai des amis qui m'aident. Je ne m'intéresse à aucune religion. Je veux que tout le monde soit heureux dans sa vie et je ne souhaite le mal de personne. J'espère rentrer dans ma patrie. Mes mère et père bien aimés sont morts. Mais qu'est-ce qu'on peut faire... le destin. J'aime la langue anglaise, je la parle ; et je veux apprendre l'allemand. La seule chose que je souhaite est que mes parents et les personnes que j'aime reviennent. Je voudrais aller chez mon frère en Allemagne et devenir neurologue parce que j'avais perdu mon calme en Syrie, j'étais tout le temps perdu. Je ne souhaite à personne de traverser ce que j'ai subi.

Extrait 1 : Exemple de texte identitaire. 
Nous tenterons de démontrer que la production de textes identitaires par des réfugiés mineurs non accompagnés peut renforcer leur estime d'eux-mêmes $(H 1)$, leur motivation pour l'apprentissage de nouvelles langues à l'école (H2) et le développement de compétences qui pourraient conduire à une meilleure intégration (aux plans affectif, linguistique, culturel et socioculturel) au sein du système scolaire du pays d'accueil transitoire ou définitif $(\mathrm{H} 3)$.

\section{Méthodologie}

Cette recherche participative porte sur une collection de textes identitaires produits en ligne ou en présentiel en 2017 par 30 réfugiés mineurs non accompagnés d'origine syrienne, afghane ou pakistanaise, âgés de 14 à 18 ans, résidant initialement au centre d'accueil de Diavata, dans la banlieue de Thessaloniki (Grèce), et dont certains ont pu finalement rejoindre des parents éloignés en Allemagne et en Belgique (réunification familiale). Au centre d'accueil de Diavata, le projet didactique dans lequel ces activités ont été proposées était peu contraignant : il s'agissait simplement, pour les animateurs et les enseignants des langues, d'organiser des activités éducatives qui leur semblaient les mieux adaptées à aider les réfugiés mineurs non accompagnés dans leur voyage et leur éventuelle installation.

Les productions et les interactions étudiées étaient publiques, publiées sur le réseau Facebook, ou privées, postées sur la messagerie Messenger. La langue en ligne se matérialise au moyen de claviers et la variabilité de la forme linguistique écrite suppose une série de fonctions pragmatiques « dans le travail de contextualisation et l'indexation des identités sociales et des relations " (Androutsopoulos, $2014: 5$ ). En suivant les réactions de leurs camarades en ligne et en interagissant avec eux, les adolescents ont pu intégrer et mieux comprendre - par le biais des outils offerts par Facebook comme, par exemple, la traduction automatique de leurs commentaires - les normes du texte identitaire et sont ainsi parvenus à produire du sens. Le dégagement de toute norme stricte d'écriture a entraîné une participation plus active. Dans ces textes, on pourrait dire que les jeunes réfugiés observés s'ouvrent à la diversité linguistique et deviennent plus curieux à propos des langues utilisées par leurs pairs (Armand et al., 2014).

La méthodologie adoptée est la même que celle de nos précédentes recherches (Cf. par exemple, auteur en collaboration, 2013) : une analyse de contenu du corpus a permis d'isoler et de recenser les représentations qu'ont les élèves de leur identité et de tout ce qui leur semble contribuer à la constitution de cette dernière. Le complètement d'une grille d'analyse (Fig. 1), construite et appliquée par deux chercheurs indépendants, a ainsi abouti à des résultats sensiblement identiques. 
Cette grille revêt la forme d'un tableau à double entrée. Les micro/macro fonctions $(C E C R, 2001)$ réalisées par les réfugiés dans leurs textes sont recensées puis portées en abscisse. Les référents $(C E C R, 2001)$ relevés sont, quant à eux, portés en ordonnée.

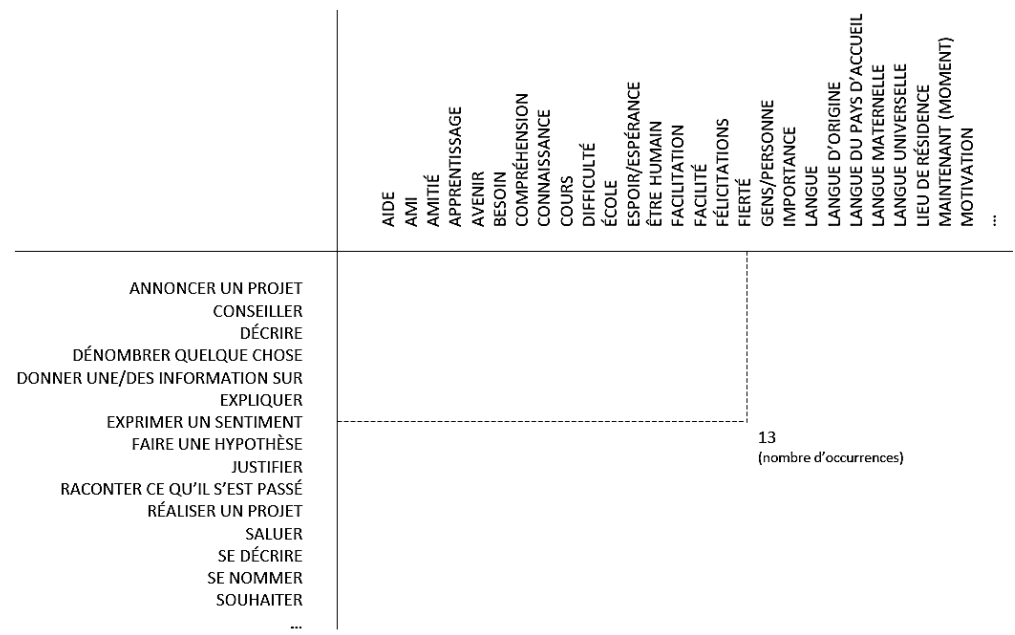

Figure 1 : Modèle de tableau permettant la mise en relation des fonctions réalisées et des référents recensés.

Une telle disposition permet - outre une indexation pratique des séquences isolées - de ne pas nous limiter au recensement des seules références aux pratiques d'apprentissage des langues et de systématiquement déterminer l'utilisation fonctionnelle (" donner des informations sur... ", " exprimer un sentiment ", " justifier » qui est faite de chacun des énoncés intégrant l'un des référents (avenir ", " fierté ", " motivation »). Ces listes de fonctions et de référents sont constituées et complétées par les chercheurs au fil de l'analyse des textes composant le corpus. Le nombre d'occurrences de chaque paire (fonction, référent) est également calculé.

\section{Résultats}

\subsection{Renforcement de l'estime de soi}

Les résultats de l'analyse, issus de la synthèse des représentations identitaires le plus souvent exprimées, sont les suivants².

${ }^{2}$ Les reproductions de fragments de textes identitaires sont toutes accompagnées d'une traduction par l'auteur, placée désormais en note, en bas de page. 
La production de textes identitaires par des réfugiés mineurs non accompagnés semble contribuer au renforcement de leur estime de soi. Le processus de la rédaction de ces textes dans un climat de confiance paraît avoir créé chez eux une sorte de fierté née des compétences acquises. La dynamique du groupe formé tout au fil de la coopération avec la tutrice a probablement joué un rôle important. On peut parler d'un contexte d'échanges fertiles à travers diverses pratiques pédagogiques et psychosociales qui ont permis l'établissement d'un climat d'interaction, de confiance, de respect mutuel.

Ainsi, les auteurs des textes identitaires ne craignent pas ou plus de présenter les grands traits de leur personnalité :

" J'ai une volonté très forte, celle de ne pas laisser tomber. »

Ils mettent en avant leur répertoire plurilingue, leur plurilinguisme, comme un atout majeur de leur identité. Une augmentation de la fréquence des échanges de pratiques plurilingues et du translangage ${ }^{3}$ - notamment l'alternance codique (Cummins, 2006) - au fil des interactions est d'ailleurs également observée au fil du temps (Extrait 2).

\footnotetext{
I. . . Jr. Hey everyone my name is Ratihb i $\mathrm{m}$ from afghanistan i live in germany before i was in greece my mother language is dari but $\mathrm{i}$ can speak urdu,english,deutsch,persian and a little ellinika melaw me ${ }^{\mathrm{\theta}}$, amd i really miss my friends from greece love you all guys -200

Mov a
}

Extrait 2 : Conscience des bienfaits du plurilinguisme. ${ }^{4}$

\subsection{Renforcement de la motivation}

La production de textes identitaires par des réfugiés mineurs non accompagnés peut renforcer leur motivation pour l'apprentissage de nouvelles langues à l'école (Extrait 3). Ils manifestent une réelle appétence pour un milieu d'apprentissage alternatif. L'école qui semble avoir une autre structure par rapport à celle de leur pays d'origine encourage plus à l'apprentissage de plusieurs langues, se présente comme plus ouverte à la diversité linguistique.

${ }^{3}$ Comme l'a noté García (2011) pour les étudiants qui utilisent le translanguage, cela devient un élément clé de la production de sens. Cette pratique permet aux bilingues émergents de relever des défis dans la compréhension et la production du langage lorsqu'ils créent leur propre texte et transmettent leurs voix uniques (Tsokalidou,2016)

${ }^{4}$ Traduction par l'auteur : «Salut à tout le monde, je m'appelle Ratihb je suis d'Afghanistan j'habite en Allemagne avant j'étais en Grèce ma langue maternelle est le dari mais je peux parler urdu, anglais, allemand, persan et un peu le grec « ellinika melaw » (translittération de la phrase grecque je parle grec) et mes amis de Grèce me manquent, je vous aime tous. » 
not

Hello everybody my name is Ahmad I am from Syria and living now in Belgium in the capital Brussels I speak Arabic and English leaming now French and Dutch and in soon future I would like to learn Greek language cause I had already experience with this language and I have a lot of great friends from there then I could talk with them in they are language $\Delta \varepsilon i \tau \varepsilon \tau \eta \mu \varepsilon \tau \dot{\alpha} \varphi \rho \alpha \sigma \eta$

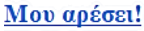

Arávtion

2

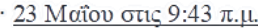

Apaipeor

12

1 .... Even here the school it's great with the teachers they are so kind in that job but also we have to be respectful for what they are doing to us the language it's little bit hard to learn it but I believe when you like or love something and you will focus on it then you gonna to got it and succeed $\Delta \varepsilon i \tau \varepsilon \tau \eta \varepsilon \tau \dot{\alpha} \varphi \rho \alpha O$

Extrait 3 : Renforcement de la motivation. ${ }^{5}$

Le profil de l'enseignant constitue un facteur encourageant pour l'apprentissage des langues. Les réfugiés ressentent l'influence positive que peuvent avoir des enseignants-modèles sur l'évolution de leurs pratiques. Selon les témoignages oraux, mais aussi dans leurs productions écrites, les adolescents ont observé une grande différence entre les profils des enseignants qui avaient d'habitude un profil autoritaire et distant vis-à-vis des élèves dans les écoles d'origine ${ }^{6}$ :

" De même, ici les écoles sont géniales avec les enseignants ; ils sont si gentils. » " Maintenant j'apprends le français et le hollandais. »

Pour les réfugiés, la difficulté de l'apprentissage des langues est occultée par les bienfaits que procurent la connaissance de ces langues (Extrait 4). Ces langues d'origines appartiennent à des branches (sous-familles) de langues différentes et/ou utilisant des alphabets différents, ce qui rend souvent l'apprentissage plus difficile. Cependant les avantages que procurent l'apprentissage de ces langues effacent les difficultés rencontrées et activent l'intercompréhension et la construction du sens par le biais d'autres mécanismes comme celui du translangage qui construit du sens compréhensible, qui favorise l'inclusion des autres et qui médiatise la compréhension à travers les groupes linguistiques (García, 2009 : 307-308).

${ }^{5}$ Trad. : « Bonjour à tous, je m'appelle Ahmad, je suis de Syrie et je vis maintenant en Belgique dans la capitale, Bruxelles. Je parle arabe et anglais, maintenant j'apprends le français et le néerlandais et dans un proche avenir je voudrais apprendre l'anglais j'avais déjà l'expérience de cette langue et j'ai beaucoup de bons amis à partir de là, alors je pourrais parler avec eux dans leur langue »

${ }^{6}$ Dans leurs témoignages, les réfugiés se réfèrent aux enseignants rencontrés en Grèce et aussi dans les pays d'accueils finaux. 
Analyse des propos tenus par des refugiés mineurs non accompagnés dans...

Awa... I It's help to connection with people and to understand each other and i like to learn everything difficult to make easy for another people they see it difficult and $i$ have many friends from different countries so $i$ like to speak with them in they are language

Extrait 4 : Effacement de la difficulté de l'apprentissage des langues. ${ }^{7}$

Les réfugiés prennent ainsi conscience du fait que l'apprentissage de la langue d'accueil à l'école constitue un facteur primordial pour une intégration dans la société (Extraits $5 \& 6$ ). Les adolescents qui, à cause de la guerre souvent, n'ont pas eu la chance d'avoir une littératie dans quelque langue (auteur, 2018, recherche en cours) présentent, d'une façon mûre et expérimentée, que les langues facilitent leur propre intégration dans la société d'accueil. Selon nos données sur le terrain, d'ailleurs, les adolescents plurilingues dans les sociétés d'accueil jouent souvent le rôle de médiateurs pour leurs pairs.

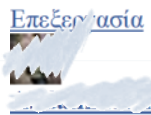
ed Hello my name is Abdallah i am from Iraq my mother tongue is Arabic and i speak English and

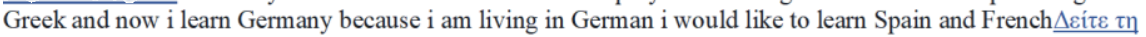

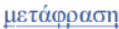

Is: Greek and I living in Germany and I learn German I want to speak French and Japanese $\Delta \varepsilon i \tau \varepsilon \tau \eta \mu \varepsilon \tau \alpha \dot{c} \rho \alpha \sigma \eta$

Extraits 5 \& 6 : Facteurs d'inclusion. ${ }^{8}$

Les réfugiés manifestent par ailleurs la volonté qu'ils ont d'affirmer leur plurilinguisme et de maîtriser de nouvelles langues (Extrait 7). Leur intention de faire des études ou d'être intégrés est étroitement liée avec l'apprentissage des langues.

« J'essaie d'apprendre l'arabe puis j'apprendrai le français, l'italien et l'allemand »

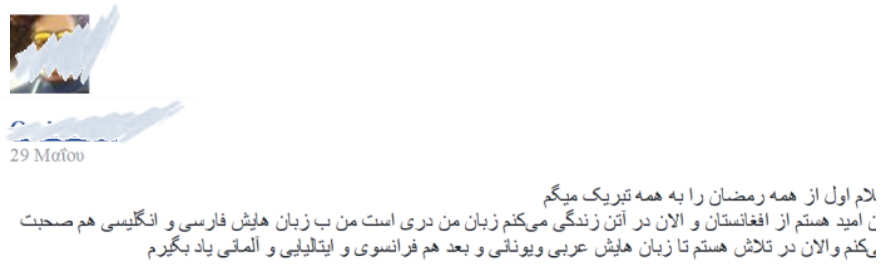

Extrait 7 : Volonté d'apprendre des langues. ${ }^{9}$

\footnotetext{
${ }^{7}$ Trad. : « Ça aide à rencontrer des personnes et à se comprendre l'un l'autre et j'aime apprendre des choses difficiles pour les rendre faciles auprès d'autres personnes qui trouvent ça difficile et j'ai plein d'amis issus de différents pays et ainsi j'aime parler avec eux dans leur propre langue » ${ }^{8}$ Trad. fidèle: «Bonjour mon nom est Abdallah je suis d'Irak ma langue maternelle est l'arabe et je parle anglais et grec et maintenant j'apprends l'Allemagne parce que je vis en allemand je voudrais apprendre l'Espagne et le français

${ }^{9}$ Trad. : « Je vous félicite au nom de tout le Ramadan
} 
Ils prennent aussi conscience du fait que certaines langues sont plus disséminées que d'autres (Extrait 8) et qu'il est important d'en tenir compte pour son avenir professionnel.

« Si tu peux parler anglais tu peux parler avec tout le monde »

se.

I Hello everyone. My name is usman $\mathrm{i}$ am from pakistan. But now $\mathrm{i}$ am in sandorini island of greece. I can speak english greek turkish punjabi urdu siraiki pashto and little bit persian. But my mother tongue is punjabi. I want to learn only English. Because If you can speak english you can speak with all world. I think in world if you go any country you can talk with this country people. I can speak british english. But $\mathrm{i}$ want to learn american english.

Extrait 8 : Intérêt des langues plus disséminées. ${ }^{10}$

\subsection{Développement de compétences inclusives}

La production de textes identitaires par des réfugiés mineurs non accompagnés peut aussi renforcer le développement de compétences qui favorisent l'inclusion dans les pays d'accueil transitoire ou définitif.

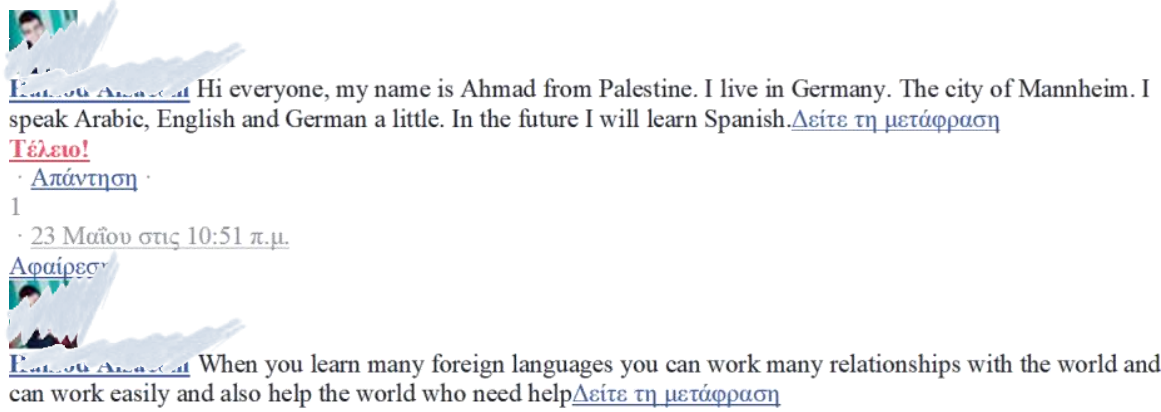

Extraits $9 \& 10$ : Facilitation de la communication. ${ }^{11}$

J'espère vivre en Afghanistan et maintenant à Athènes Ma langue est le dari Je parle anglais et anglais dans mes propres langues J'essaie d'apprendre les langues arabes à Vinny et ensuite le français, l'italien et l'allemand.»

${ }^{10}$ Trad. : « Bonjour à tous. Mon nom est Usman je suis du Pakistan. Mais maintenant je suis à I'île de Santorin en Grèce. Je peux parler anglais grec turc punjabi urdu siraiki pashto et peu peu persan. Mais ma langue maternelle est le punjabi. Je veux apprendre seulement l'anglais. Parce que si vous pouvez parler anglais, vous pouvez parler avec tout le monde. Je pense que dans le monde si vous allez n'importe quel pays, vous pouvez parler avec les gens de ce pays. Je peux parler l'anglais britannique. Mais je veux apprendre l'anglais américain. »

${ }^{11}$ Trad. : " Bonjour à tous, je m'appelle Ahmad de Palestine. Je vis en Allemagne. La ville de Mannheim. Je parle l'arabe, l'anglais et un peu l'allemand. Dans l'avenir, j'apprendrai l'espagnol. 
Les langues apprises jettent un pont entre les gens qui permet l'établissement de la communication (Extrait 9 \& 10).

Les langues apprises constituent une aide pratique et favorisent la compréhension mutuelle. Les représentations du plurilinguisme sont toutes très positives (Extrait 11). Ces langues, recensées lors de la composition des répertoires de sujets étudiés, peuvent contribuer à la création de sens. Ces stratégies variées peuvent être considérées comme un processus ou une réalité potentielle et dynamique susceptible de développer ou d'améliorer les aptitudes et les compétences plurilingues qui accompagneront des jeunes tout au long de leur vie.

\section{d It's help to connection with people and to understand each other and i like to learn everything difficult to make easy for another people they see it difficult and $i$ have many friends from different countries so $i$ like to speak with them in they are language}

Extrait 11 : Représentations positives du plurilinguisme. ${ }^{12}$

La connaissance d'autres langues permet aux réfugiés de présenter leur bagage socioculturel, des valeurs importantes dans leur culture d'origine. En d'autres termes, les réfugiés peuvent mettre en avant leurs identités. Ces identités sont également enrichies (Spotti, 2011). Les mots ont donc une charge idéologique (Rampton, 2005) puisqu'ils sont soumis aux valeurs en jeu, au moment et dans l'espace où ils sont prononcés (Blommaert, 2005). Ainsi, des productions écrites des adolescents, émerge une réflexion sur les valeurs culturelles qui composent justement cette charge idéologique. La langue constitue donc un lien avec la culture du pays d'origine, mais aussi l'élément de base de la préservation d'une identité particulière.

(i)

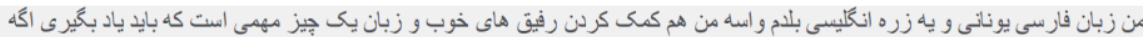

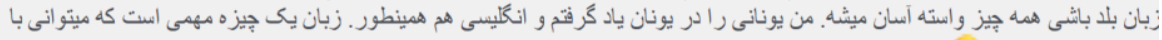

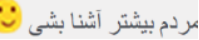

Extrait 12 - Familiarisation avec l'Autre ${ }^{13}$

Quand on apprend beaucoup de langues, on peut créer beaucoup de relations dans le monde et on peut travailler facilement et aussi aider les gens qui en ont besoin »

12 Trad. : « Ça aide à rencontrer des personnes et à se comprendre l'un l'autre et j'aime apprendre des choses difficiles pour les rendre faciles auprès d'autres personnes qui trouvent ça difficile et j'ai plein d'amis issus de différents pays et ainsi j'aime parler avec eux dans leur propre langue $"$.

${ }^{13}$ Trad. : « Si vous parlez la langue, tout devient facile. J'ai également appris le grec en Grèce et l'anglais. La langue est une chose importante que vous pouvez faire, pour mieux connaître les gens ». 
L'usage d'autres langues permet aussi que se développe l'empathie, la familiarisation avec l'Autre (Extrait 12). Une sorte de biographie langagière (Thamin \& Simon, 2009) qui est discernable dans les productions exprime la prise de conscience par les réfugiés du fait que le répertoire plurilingue constitue une source de richesse et un outil de contact avec les autres. Une identité toujours en évolution est présentée, une identité composée de plusieurs appartenances : l'appartenance religieuse et l'appartenance linguistique qui sont, à l'évidence, parmi les plus puissants constituants d'une identité. Mais ils fonctionnent différemment, et se trouvent parfois en concurrence : I'appartenance religieuse est exclusive, l'appartenance linguistique ne l'est pas (Tsokalidou \& auteur, 2009). Le développement de I'appartenance linguistique et culturelle, par le biais de ces textes pourrait contribuer à réduire les tensions au sein des sociétés européennes comme dans le reste du monde.

\section{Discussion}

La production de textes identitaires par des réfugiés mineurs et non accompagnés peut donc bien renforcer

- l'estime qu'ils ont d'eux-mêmes, l'affirmation sous un jour gratifiant de leur personnalité, de leurs valeurs, de leur plurilinguisme (H1) ;

- leur motivation pour l'apprentissage de nouvelles langues à l'école, la conscience des bienfaits de la connaissance des langues, la prise de conscience du facteur d'inclusion que constitue l'apprentissage des langues $(\mathrm{H} 2)$;

- le développement de compétences qui pourraient conduire à une meilleure intégration au sein du système scolaire du pays d'accueil par la construction d'un pont de communication, l'intercompréhension et l'empathie (H3).

Le texte identitaire constitue donc un outil prêt à l'emploi, mais pour peu que soit instauré un climat de confiance entre acteurs de l'éducation. Comment créer de telles conditions ? Sommes-nous prêts, en tant qu'écoles européennes, à accueillir ou à intégrer ce vaste répertoire des langues évoqué ? Comment les didactiques du plurilinguisme pourraient-elles contribuer à cette intégration ?

Les résultats de notre étude peuvent inciter les enseignants de langues, mais aussi peut-être l'École dans sa globalité ainsi que les organisations non gouvernementales à observer puis à ancrer les compétences linguistiques et culturelles des réfugiés nouvellement arrivés, afin qu'ils trouvent plus facilement leur place dans la société qui les accueille.

Une étude des processus d'articulation des identités chez les mineurs réfugiés sera très utile pour leur intégration dans l'éducation institutionnelle, en Grèce 
ou ailleurs. La diffusion de nos résultats auprès dedes enseignants, des formateurs des enseignants et des décideurs peut sensibiliser à des questions telles que celles de l'interaction entre les langues, du plurilinguisme et des pratiques de translangage (García, 2009), en tant que ressources utiles pour l'apprentissage et l'enseignement. Le texte identitaire quelle que soit sa forme nous incite à mieux prendre conscience de la nécessité croissante de la découverte des identités plurielles qui nous entourent, la nécessité cruciale de l'ouverture vers l'autre.

\section{BIBLIOGRAPHIE}

Act!onaid, Care, Norwegian Refugee Council, Rescue, International Federation of Red Cross and Red Crescent Societies, Danish Refugee Council, Jesuit Refugee Service, Save the Children, Solidarity Now, Oxfam, Translators without Borders (2016), More than Six Months Stranded - What Now? A Joint Policy Brief on the Situation for Displaced Persons in Greece. Rapport sans sauteurs. Repéré à http://www.statewatch.org/news/2016/oct/greece-M ore-than-Six-Months-Stranded-What-Now.pdf

Armand Fr., Combes E., Boyadjiéva G., Petreus M. et Vatzlaaroussi M. (2014), Écrire en langue seconde: les textes identitaires plurilingues (dans) "Québec français", n 173, p. 25-27.

Androutsopoulos J. (2014), Moments of sharing: Entextualization and linguistic repertoires in social networking (dans) "Journal of Pragmatics", $n^{\circ}$ 73, p. 4-18.

Bernaus M., Andrade A.I., Kervran M., Murkowska A. et Trujillo Sáez F. (2007), Kit de formation. Strasbourg/Graz : Conseil de I'Europe / Centre européen pour les langues vivantes.

Blommaert J. (2005), Discourse. Cambridge: Cambridge University Press.

Candelier M. et al. (2012), Le CARAP : Un Cadre de Référence pour les Approches Plurielles des Langues et des Cultures : Compétences et ressources. Strasbourg : Conseil de l'Europe. Repéré à : http://carap.ecml. at/ [consulté le 05.01.2018].

Cummins J., Bismilla V., Chow P., Giampapa F., Leoni L. et Sandhu P. (2006), ELL Students Speak for Themselves: Identity Texts and Literacy Engagement in Multilingual Classrooms, Repéré à : http://www.curriculum.org/secretariat/files/ELLidentityTexts.pdf [consulté le 05.01.2018].

Cummins J. (2009), Transformative Multiliteracies Pedagogy: School-Based Strategies for Closing the Achievement Gap (dans) "Multiple Voices for Ethnically Diverse Exceptional Learners", n 11(2), p. 38-56. 
Delhaye O. et Gatsi G. (2013), Bonnes pratiques méthodologiques pour renforcer l'estime de soi des élèves migrants dans une école primaire en Grèce (dans) "Éducation et sociétés plurilingues" n³4, p. 55-68.

De Bonville J. (2000), L'analyse de contenu des médias : de la problématique au traitement statistique. Paris-Bruxelles : De Boeck-Université.

Deprez C. (1996), Parler de soi, parler de son bilinguisme, entretiens autobiographiques et récits de vie d'apprenants et de bilingue, (dans) "AILE", $\mathrm{n}^{\circ}$ 7, p. 155-180.

Deprez C. (2000), Histoires de langues, histoires de vies (dans) "Cahiers de sociolinguistique", $n^{\circ} 5$, p. 167-174.

García O. (2009), Bilingual education in the 21st Century: A global perspective. Oxford: Wiley-Blackwell

García O. (2011), Educating New York's bilingual children; constructing a future from the past (dans) "International Journal of Bilingual Education and Bilingualism", $n^{\circ} 14,2:$ p. 133-153.

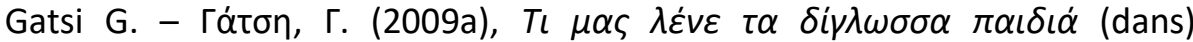

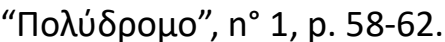

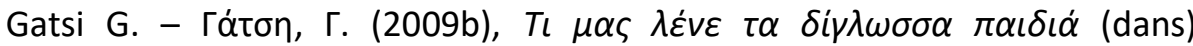

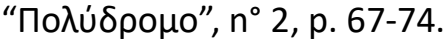

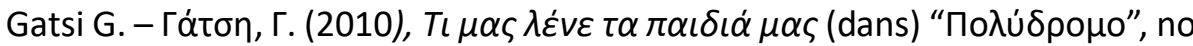
3, p. 92-97.

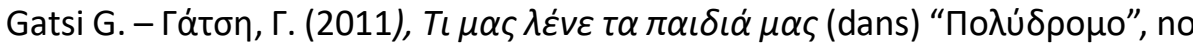
3, p. 79-82.

Gatsi G. (2017), Contribution du texte identitaire au renforcement de l'identité plurilingue et à l'accroissement de la motivation pour l'apprentissage $d^{\prime}$ 'une nouvelle langue à l'école (dans) "Revue Méthodal", $\mathrm{n}^{\circ} 1$. Repéré à https://revue.methodal.net/?article97_[consulté le 05.01.2018].

Gatsi G. et Delhaye O. (2015), Représentations de l'identité pluriculturelle et épanouissement scolaire : aux "pays magique des plurilingues » (dans) "Migration et société", $\mathrm{n}^{\circ} 162$, p. 121-138.

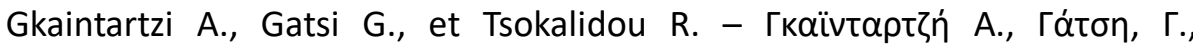

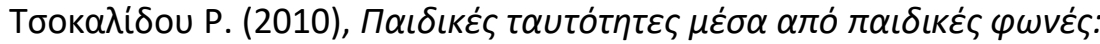

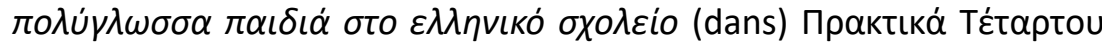

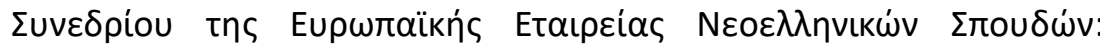

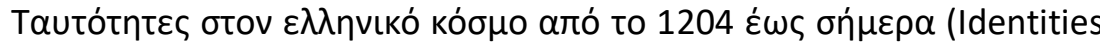

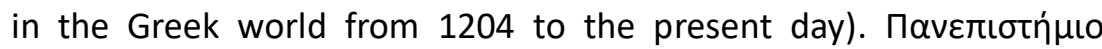
Граvádoc, 9-12/09/2010.

Haut-Commissariat des Nations unies pour les réfugiés (2017). Rapport du Centre national pour la solidarité sociale. Situation actuelle des mineurs 
non accompagnés en Grèce. Repéré à https://data2.unhcr.org/en/documents/download/61145

Rampton B (2005), Crossing: Language and ethnicity among adolescents. Manchester: St. Jerome.

Spotti M. (2011), Modernist language ideologies, indexicalities and identities: Looking at the multilingual classroom through a post-Fishmanian lens (dans) "Applied Linguistics Review”, n² 2, (2), p. 29-50.

Thamin N. et Simon D.-L. (2009), Réflexion épistémologique sur la notion de biographies langagières (dans) "Carnets d'Ateliers de Sociolinguistique" (CAS), n 4 (Praticiens et chercheurs à l'écoute du sujet plurilingue, réflexivité et interaction biographique en sociolinguistique et en didactique), E. Huver et M. Molinié (dir).

Tsokalidou R. (2016), Beyond language borders to translanguaging within and outside the educational context. Bilingualism: Cultural Influences, Global Perspectives and Advantages/Disadvantages, p. 108-118.

Tsokalidou R. et Gatsi G. (2009), Questions de langue et d'identité : le cas $d^{\prime}$ Amin Maalouf (dans) "Synergies", n’ 2, p. 195-202. 